\title{
Training Based Channel Estimation for Multitaper GFDM System
}

\author{
Shravan Kumar Bandari, ${ }^{1}$ Venkata Mani Vakamulla, ${ }^{1}$ and A. Drosopoulos ${ }^{2}$ \\ ${ }^{1}$ Electronics \& Communication Engineering, National Institute of Technology, Warangal 506004, India \\ ${ }^{2}$ Electrical Engineering, TEI of Western Greece, 26334 Patras, Greece \\ Correspondence should be addressed to Shravan Kumar Bandari; shravnbandari@gmail.com
}

Received 27 January 2017; Revised 27 July 2017; Accepted 20 August 2017; Published 1 October 2017

Academic Editor: Jian Qiao

Copyright (c) 2017 Shravan Kumar Bandari et al. This is an open access article distributed under the Creative Commons Attribution License, which permits unrestricted use, distribution, and reproduction in any medium, provided the original work is properly cited.

\begin{abstract}
Recent activities in the cellular network world clearly show the need to design new physical layer waveforms in order to meet future wireless requirements. Generalized Frequency Division Multiplexing (GFDM) is one of the leading candidates for $5 \mathrm{G}$ and one of its key features is the usage of circular pulse shaping of subcarriers to remove prototype filter transients. Due to the nonorthogonal nature of the conventional GFDM system, inherent interference will affect adversely channel estimation. With Discrete Prolate Spheroidal Sequences (DPSSs) or multitapers as prototype filters an improved orthogonal GFDM system can be developed. In this work, we investigate channel estimation methods for multitaper GFDM (MGFDM) systems with and without Discrete Fourier Transform (DFT). The simulation results are presented using Least Squares (LS) and Minimum Mean Square Error (MMSE) channel estimation (CE) methods. DFT based CE methods provide better estimates of the channel but with an additional computational cost.
\end{abstract}

\section{Introduction}

The basic idea behind the multicarrier technique was to divide the total available bandwidth into a number of subbands, allowing the handling of channel effects in an efficient manner. Each generation of mobile communication systems offered many services after which these systems are classified. There are enormous capability advancements in the mobile communications from $1 \mathrm{G}$ to the current $4 \mathrm{G}$. The upcoming future generation, $5 \mathrm{G}$, is expected to have an even better coverage area capability, high data connectivity of $1 \mathrm{Gbps}$, less energy consumption, high security, and better spectral utilization. Moreover, emerging applications such as self-driving cars, real time gaming, and industrial automation control demand a low latency period of the order of less than $1 \mathrm{~ms}$. Even though there is no standard yet for $5 G$, the industrial and academic research communities are exploring a number of possible implementation options.

A widely used air interface multicarrier (MC) technique in many present wireless standards is Orthogonal Frequency Division Multiplexing (OFDM). Some of the standards include Wireless Local Area Networks (WLAN 802.11), 4GLTE (Long Term Evolution) Advanced, Digital Video Broadcasting for Terrestrial Television (DVBT), and Digital Audio Broadcasting (DAB) [1]. OFDM gains its popularity due to its efficient implementation with Fast Fourier Transform (FFT)/Inverse Fast Fourier Transform (IFFT) signal processing blocks and its robustness to intersymbol interference (ISI) with simple low complexity equalization. Despite its advantages, in the area of future vision applications, OFDM suffers from a number of drawbacks, such as high Peak to Average Power Ratio (PAPR), addition of cyclic prefix (CP) per symbol that reduces bandwidth efficiency, high out-of-band (OOB) emission due to the use of rectangular filters, and high synchronisation issues due to orthogonality mismatch.

A great deal of effort has been expended in the search for an alternate multicarrier waveform to serve future generation, 5G. Some of the possible contenders with a variety of properties are Filter Bank Multicarrier (FBMC) [2], Universal Filtered Multicarrier (UFMC) [3], and GFDM (Generalized Frequency Division Multiplexing) [4]. More insights into each waveform and their individual properties, such as 
spectral efficiency and bit error rates (BER), can be found in the literature and references therein.

GFDM [4] is a flexible multicarrier technique in which the data is divided into a number of subcarriers and subsymbols, using circular pulse shaping for each subcarrier. GFDM covers OFDM as a special case, retaining the advantages of OFDM and dealing with the limitations. For example, GFDM uses one cyclic prefix $(\mathrm{CP})$ per group of subsymbols and prototype filtering with low $\mathrm{OOB}$ emission to improve bandwidth utilization. However, due to the nonorthogonal nature of conventional GFDM, channel estimation is not straightforward.

Generally, the channel impulse response can be estimated using training sequences or pilot symbols. Reference [5] introduces two scattered pilot based channel estimation methods for GFDM, Pilot Interference Cancellation (IC) and Transmitter IC. The article gives an insight into interference cancellation at either the transmitter or the receiver of the communication system. Blind estimation of channel block duration, symbol duration, and number of subcarriers are discussed in [6]. Filters with low OOB are desirable for GFDM systems which is quite challenging. In [7], the authors introduce multitapers as prototype filters in order to improve the orthogonality of a conventional GFDM system, naming the result as multitaper GFDM (MGFDM). Another advantage of using multitapers is the low $\mathrm{OOB}$ emission which in turn will increase the spectral efficiency. Reference [8] presented the basic framework of how GFDM is used for physical layer services of $5 \mathrm{G}$ networks. In this context as multitaper GFDM is the modified version of the conventional GFDM waveform, where we use tapers to pulse-shape each subcarrier instead of circular pulse shaping as in GFDM, MGFDM is related to $5 \mathrm{G}$ networks and can be regarded as a potential candidate for $5 \mathrm{G}$ networks.

OFDM channel estimation is addressed by [9] based on time domain channel statistics. Channel estimation for various pilot patterns is discussed in [10] and references therein. A great amount of research covering channel estimation for OFDM has been done over the years and much of it can be modified and applied to GFDM systems.

However the subcarrier orthogonality which exists in OFDM is no longer valid for GFDM, as it uses pulse shaping for the subcarriers. There are very few papers available in the literature for GFDM signal channel estimation [5] and to the best of the authors knowledge there is no literature covering channel estimation using Least Squares (LS) and Minimum Mean Square Error (MMSE) and in combination with DFT to multitaper GFDM. These methods are well discussed in the past for various multicarrier signals. However, as multitaper GFDM is the most advanced modulation scheme using a sophisticated block based structure, implementing the above estimation algorithms under severe channel conditions is a difficult task. It should also be noted that any real-life implementation of a wireless system will include a method of channel equalization to improve performance and therefore requires a way to do a running channel estimation to handle effectively changing channel conditions.

Motivated by the above facts, therefore, in this paper, pilot symbol based channel estimation (CE) is carried out for the MGFDM system model. This approach is applicable in general, to any multicarrier technique. Pilot symbols are multiplexed along with the information data symbols. These pilot symbols are known to both transmitter and receiver and a variety of interpolation techniques can be employed to estimate the channel response. As is widely done for $\mathrm{CE}$ when training symbols are available, the techniques used are Least Squares (LS) and Minimum Mean Square Error (MMSE).

The article is organized as follows: Section 2 gives a brief introduction about the MGFDM system model. Channel estimators under consideration are discussed in Section 3. Results are discussed in Section 4, followed by conclusions in Section 5.

\section{System Model}

GFDM is a two-dimensional multicarrier technique in which the data samples are divided among the time and frequency domains [4]. The time and frequency domain plots of root raised cosine (RRC), first DPSS, and PHYDYAS prototype filters are shown in Figure 1. Note, from the plot, that multitapers have deeper sidelobes compared to other pulse shaping filters, which leads to an overall improved system performance [7]. In this section we will give a brief introduction on the MGFDM transceiver system model. Figure 2 shows the system model for training based channel estimation for the MGFDM system.

2.1. MGFDM Signal Model. The binary data generated from the source are grouped together to form P-QAM modulated data, where $P=2^{\mu}$ and $\mu$ is the modulation index. The training symbols or pilot information bits are inserted at an equal spacing between data symbols. The time domain signal after passing through the MGFDM modulator is given by [7]

$$
x(n)=\sum_{k=0}^{K-1} \sum_{m=0}^{M-1} d_{k}(m) g_{k, m}(n) \quad n=0,1, \ldots, K M-1,
$$

where $k=0,1, \ldots, K-1$ denotes the frequency index, $m=$ $0,1, \ldots, M-1$ denotes the subsymbol index, and $n$ is the sample index. $g_{k, m}(n)=g_{m}(n) e^{j 2 \pi k n / K}$ with $g_{m}(\cdot)$ corresponding to the $m$ th taper of length $K M$ is chosen from the Discrete Prolate Spheroidal Sequences (DPSSs) [11]. The exponential term is the frequency shifted version of the prototype filter $g_{m}(\cdot) \cdot d_{k}(m)$ is the data symbol on the $k$ th subcarrier and $m$ th subsymbol. It is important to note that MGFDM also covers OFDM with $M=1$ and $g[n]=1 / \sqrt{K}$ and Single Carrier Frequency Domain Equalization (SC-FDE) with $K=1$ and $g[n]=\delta[n]$, as special cases.

In matrix notation equation (1) can be written as [4]

$$
\mathbf{x}=\text { Ad, }
$$

where $\mathbf{A}$ is the transmitter modulation matrix as described in [12]. Matrix A incorporates all the signal processing steps, while $\mathbf{d}$ is a vector containing the data symbols $d_{k}(m)$.

Guard interval bits are added at the front side of the symbol in order to prevent intersymbol interference that may possibly affect the MGFDM system. The signal is then 

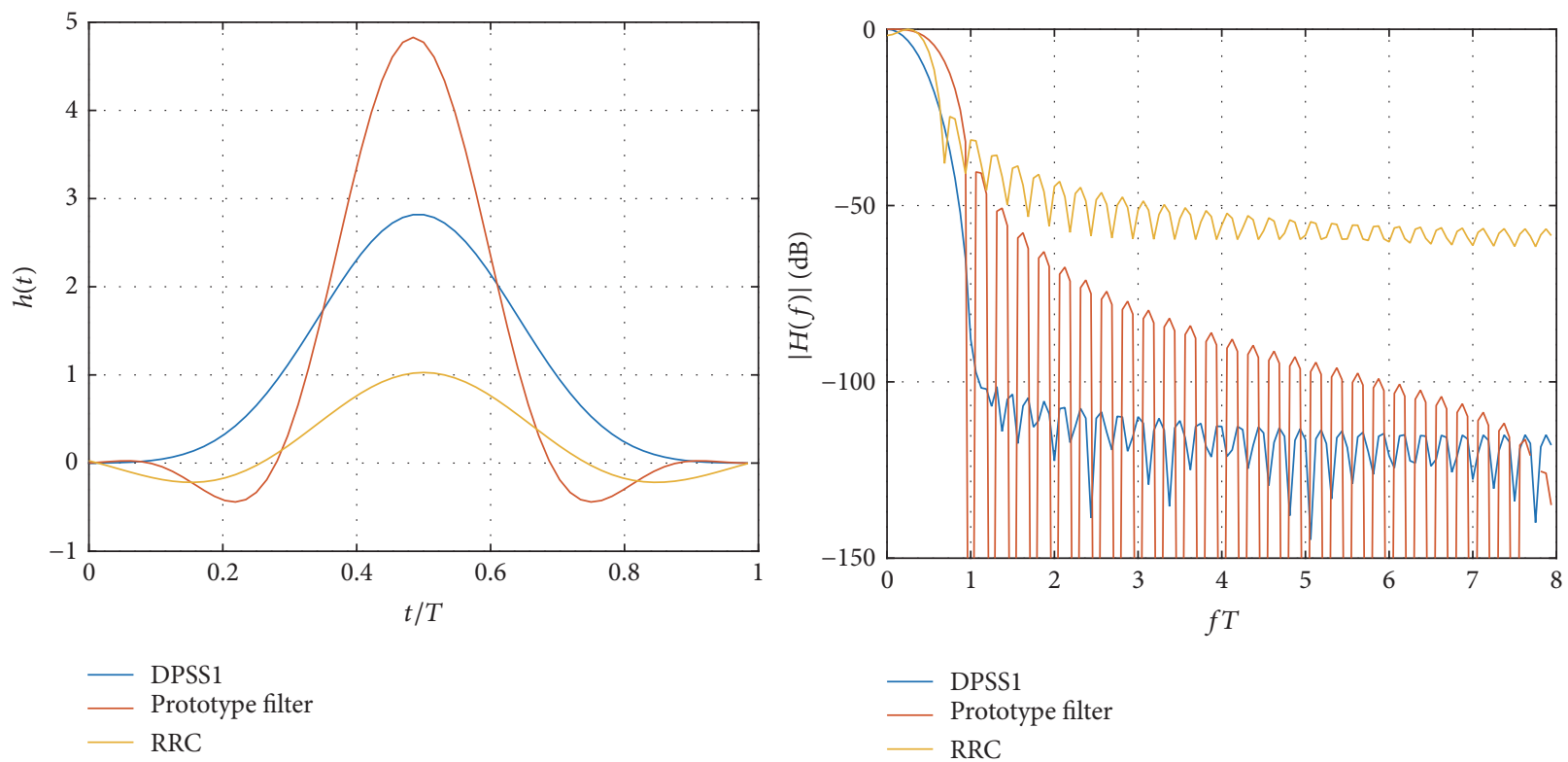

FIGURE 1: Time and frequency domain plots of RRC, DPSS1, and the PHYDYAS prototype filter.

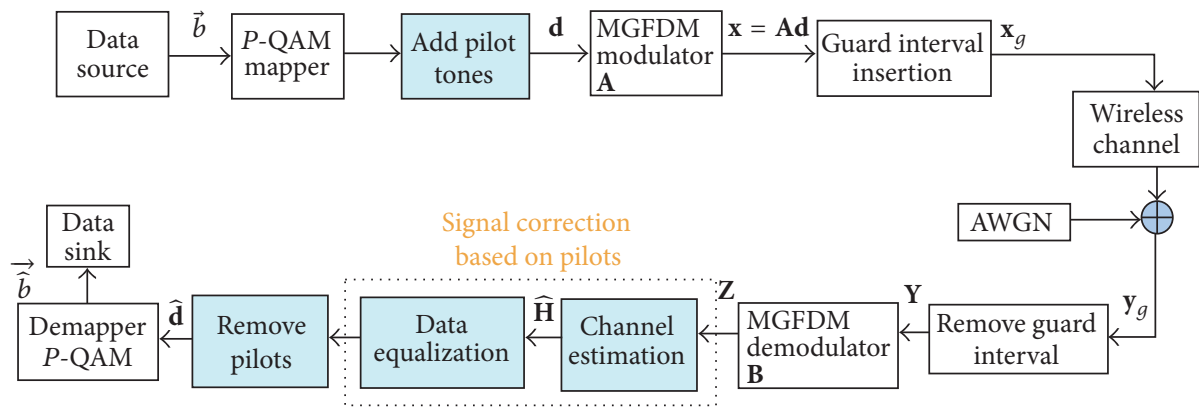

Figure 2: Baseband model for pilot based MGFDM system.

transmitted through the frequency selective multipath fading channel $h(n)$. The signal received can be represented as [5]

$$
y_{g}[n]=x_{g}[n] * h[n]+w[n],
$$

where $*$ denotes linear convolution, $h[n]$ represents the channel impulse response, and $w[n]$ is Additive White Gaussian Noise (AWGN).

The guard interval bits are removed from the corrupted signal with AWGN, that is, $y_{g}[n]$, and are sent to the MGFDM demodulator block. There are several receivers found in the literature to demodulate the data [4]. In this article we have used the zero forcing receiver given by

$$
\mathbf{B}=\left(\mathbf{A}^{\mathbf{H}} \mathbf{A}\right)^{-1} \mathbf{A}^{\mathbf{H}}
$$

B is known as the MGFDM demodulation matrix, which incorporates the reverse signal processing steps involved at the transmitter side (MGFDM modulator). The demodulated output is written as

$$
\mathbf{Z}=\mathbf{B Y}
$$

where $\mathbf{Y}$ is the received data after removal of the guard interval. It should be noted that the length of the guard interval is required to be greater than the length of the channel impulse response in order to avoid intersymbol interference. The demodulated output is passed through the signal correction block which is done based on the pilot symbols that are added at the transmitter side. The steps involved in this block are elaborated in Figure 3. In this block, the channel estimation of the pilot tones is first carried out, followed by channel interpolation (linear/spline). Finally, the demodulated data are equalized with the estimated channel coefficients.

\section{Channel Estimator}

In this section we will briefly discuss the most popularly used channel estimation techniques, Least Squares (LS) and Minimum Mean Square Error (MMSE), applicable to any multicarrier technique. This work was inspired by [13] which was originally proposed for OFDM systems. As MGFDM is the generalization of FDM systems, we carried out the same approach to estimate the channel response. 


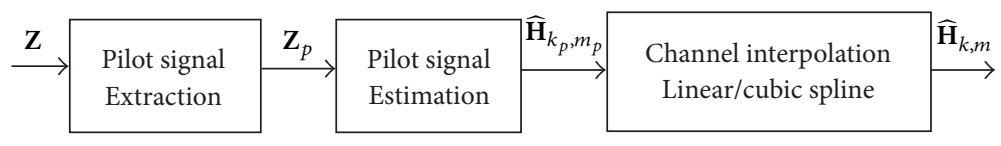

FIgURE 3: Steps involved in channel estimation.

3.1. LS. Let $\mathbf{H}_{p}$ be the channel response of the pilot symbols and $Z_{p}$ be the received pilot signal vector. Based on the LS approach [13], the channel estimates of the pilot signals are given by

$$
\begin{aligned}
\widehat{\mathbf{H}}_{p, \mathrm{LS}} & =\mathbf{X}_{p}^{-\mathbf{1}} \mathbf{Z}_{p} \\
& =\left[\frac{Z_{p}(0)}{X_{p}(0)}, \frac{Z_{p}(1)}{X_{p}(1)}, \ldots, \frac{Z_{p}\left(N_{p}-1\right)}{X_{p}\left(N_{p}-1\right)}\right],
\end{aligned}
$$

where $N_{p}$ is the number of the pilot symbols used; $\mathbf{X}_{p}$ and $\mathbf{Z}_{p}$ are the pilot symbols data at the transmitted side and the pilot information obtained at the receiver side after demodulation, respectively. Let us state that the FFT of (2) is given by

$$
X[k, m]=\operatorname{FFT}(\mathbf{x}) .
$$

Then the MSE of the $k$ th subcarrier and $m$ th subsymbol is given by

$$
\operatorname{MSE}_{\mathrm{LS}}[k, m]=\frac{\beta}{\mathrm{SNR}},
$$

where the factor $\beta$ is given by $\beta=E\left[|X[k, m]|^{2}\right] E\left[|X[k, m]|^{-2}\right]$ and is a constant depending on the constellation. For example, $\beta$ for $16-\mathrm{QAM}$ is $17 / 9$ and $\beta=1$ for BPSK [14]. SNR = $E\left[|X[k, m]|^{2}\right] / \sigma_{w}^{2}$ is the average SNR.

3.2. MMSE. Let us consider the LS solution obtained in the above approach. The MMSE estimate of the channel is given by [10]

$$
\widehat{\mathbf{H}}_{\mathrm{mmse}}=\mathbf{R}_{\mathbf{H}_{p} \mathbf{H}_{p, \mathrm{LS}}}\left(\mathbf{R}_{\mathbf{H}_{p} \mathbf{H}_{p}}+\sigma_{w}^{2}\left(\mathbf{X}_{p} \mathbf{X}_{p}^{\mathbf{H}}\right)^{-1}\right)^{-1} \widehat{\mathbf{H}}_{\mathrm{LS}},
$$

where $\mathbf{R}_{\mathbf{H}_{p} \mathbf{H}_{p, \mathrm{LS}}}=E\left[\mathbf{H}_{p} \mathbf{H}_{p, \mathrm{LS}}^{\mathbf{H}}\right]$ is the cross correlation matrix between $\mathbf{H}_{p}$ and $\mathbf{H}_{p \text {,LS }} \cdot(\cdot)^{\mathbf{H}}$ is the Hermitian operator of a matrix, $\widehat{\mathbf{H}}_{\mathrm{LS}}$ is the least square channel estimation, and $\sigma_{w}^{2}$ is the variance of the noise.

3.3. Channel Interpolation. To estimate the complete response of the channel, we make use of the interpolation concept. In this, the samples of the channel response are interpolated according to the estimated channel response of the pilot tones. This kind of work was considered for OFDM systems [13] and is shown to be efficient. In order to increase the overall system performance of such an advanced multicarrier technique (MGFDM), the complete channel state/coefficients should be estimated more accurately. Hence, information about the channel at both pilot and nonpilot locations is essentially what is required to enhance performance. The interpolation method is an efficient way to estimate the nonpilot (data location) channel characteristics by making use of the channel coefficients at the pilots. In this article we examined two interpolation techniques, namely, piecewise linear interpolation and cubic spline interpolation. For more details on these techniques, interested readers can refer to [13].

3.4. DFT Based Channel Estimation. In order to improve the performance of LS and MMSE, a DFT based channel estimation is considered. The performance of such DFT based channel estimators is analyzed for OFDM systems and $[14,15]$ provide more insights into the method. The scope of the paper is to investigate such estimators for MGFDM system using Mean Square Error (MSE) as a measuring parameter. Even though all existing methods of channel estimation techniques are directly applicable to GFDM, if the pilot symbols require a simpler and different orthogonal demodulation approach, this should be taken into account at the receiver; otherwise it might be questionable to detect pilot symbols due to the nonorthogonality nature of GFDM and pulse shaping property in MGFDM. Our implementation uses the same demodulation approach to both pilot and data symbols and we do not observe the above problem. A simple block diagram of DFT based CE is shown in Figure 4. In this, the effect of noise outside the channel delay $(L)$ is eliminated. Let $\widehat{\mathbf{H}}_{k, m}$ denote the estimation of the channel response at the $k$ th subcarrier and $m$ th subsymbol, calculated by using either the LS or the MMSE method. This is a two-step process. First, we take the IDFT of the $\widehat{\mathbf{H}}_{k, m}$ and ignore the coefficients outside the maximum channel delay. Next, the obtained time domain channel estimation coefficients are converted back to frequency domain by taking an $N$ point DFT. This can be mathematically represented as follows:

$$
\begin{aligned}
\operatorname{IDFT}\left[\widehat{\mathbf{H}}_{k, m}[\mathbf{n}]\right] & =\widehat{h}_{k, m}[n]=\widehat{h}_{\mathrm{DFT}, k, m}[n], \\
\widehat{\mathbf{H}}_{\mathrm{DFT}, k, m}[\mathbf{n}] & =\operatorname{DFT}\left[\widehat{h}_{\mathrm{DFT}, k, m}[n]\right],
\end{aligned}
$$

where $n=0,1, \ldots, K M-1$.

The individual MSE of the DFT based channel estimation is given by

$$
\operatorname{MSE}_{\mathrm{DFT}}[k, m]=\frac{L}{N} \frac{\beta}{\mathrm{SNR}},
$$

where $N$ is a constant and $L$ depends on the channel environment. According to [16], $L / N=T_{G} / T_{s}$ in IEEE 802.11 and IEEE 802.16 standards is selected from $\{1 / 32,1 / 16,1 / 8,1 / 4\}$.

The simulated MSE is given by the average of the error matrix:

$$
\mathrm{MSE}=\frac{1}{K M} E\left\{(\widehat{\mathbf{H}}-\mathbf{H})(\widehat{\mathbf{H}}-\mathbf{H})^{T}\right\}
$$




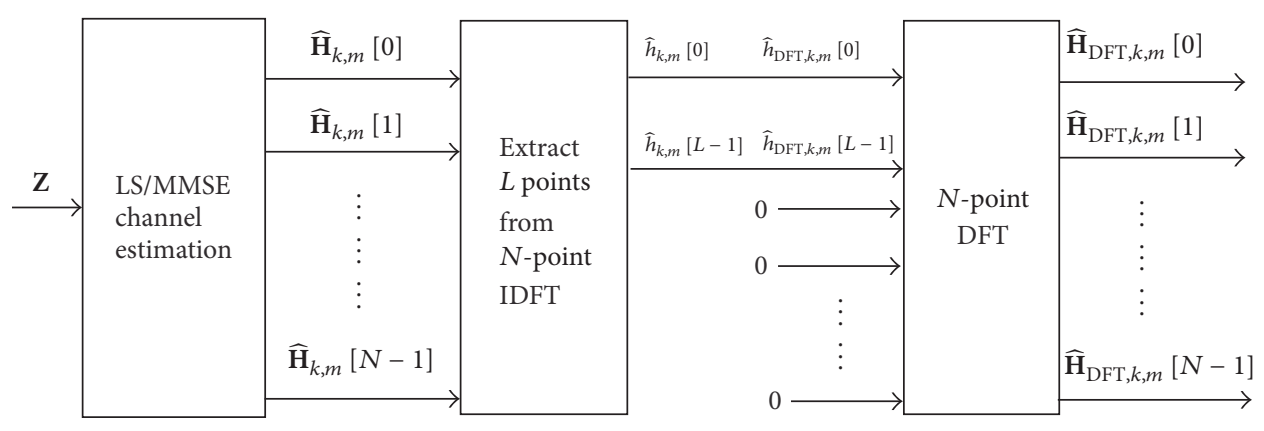

FIGURE 4: Block diagram of DFT based channel estimation.

TABLE 1: Simulation parameters.

\begin{tabular}{lc}
\hline Description & Value \\
\hline Number of subcarriers $K$, subsymbols $M$ & 32,4 \\
Total number of samples, $N=K * M$ & 128 \\
Length of CP, $N_{\mathrm{cp}}$ & 8 \\
Modulation order, $\mu$ & 4 \\
Length of pilot symbols, $N_{p}$ & 16 \\
Length of the channel, $N_{\mathrm{ch}}$ & 2 \\
\hline
\end{tabular}

where $E(\cdot)$ is the expectation operator, $(\cdot)^{T}$ is the transpose of the matrix, and $\widehat{\mathbf{H}}$ is the channel estimate.

\section{Results and Discussions}

In this section, we will discuss the performance of the channel estimation methods that are investigated in this article for the MGFDM system. We have considered a 2-tap random channel model. In Matlab we use the interp1() command for interpolation, as this command is based on piecewise linear and cubic interpolation. The simulation parameters are considered are as shown in Table 1 . We have used a total of $N$ samples in which $N_{p}$ number of pilots are inserted in between the data points with a pilot spacing of 8 .

Figure 5 shows the channel estimates that are obtained by using various types of channel estimation methods and are compared with the true channel. We have assumed that the guard interval is greater than the channel delay $L$. From the figure it is clear that the MMSE estimation shows an improvement compared to that of the LS approach. In the LS estimate we have used linear interpolation and cubic spline interpolation methods to estimate the complete channel response.

A DFT based channel estimation method using LS and MMSE is shown in Figure 6. A good match between the true channel and the estimated channel response is observed. Comparing Figures 5 and 6, an improvement is observed in estimating the channel using DFT based channel estimation.

Figure 7 illustrates the MGFDM system performance in terms of MSE for different SNR values. The MSE of the proposed three techniques with and without the use of DFT based channel estimation is provided. To strengthen the simulated results, theoretical plots for the expressions
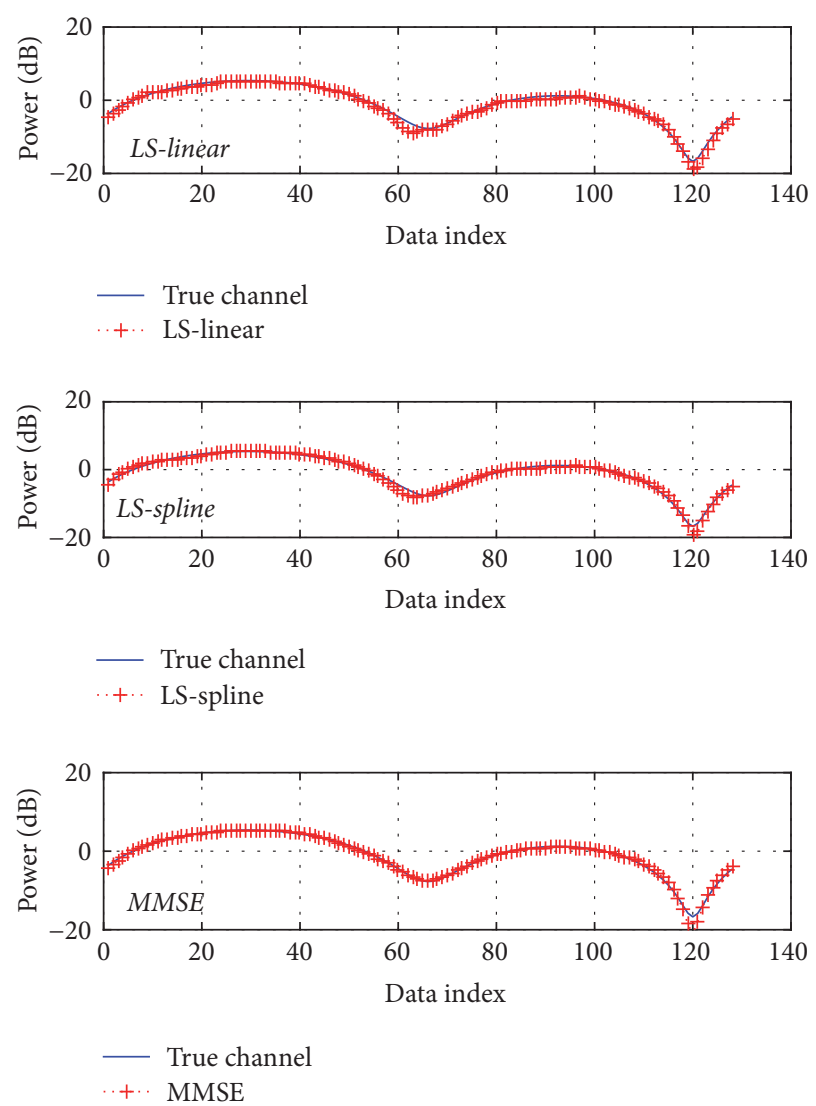

Figure 5: Channel estimation without DFT.

provided in the above sections under LS and DFT based channel estimation methods are also given. It should be noted that both simulated and theoretical values are in good agreement and match almost at every SNR. MMSE results in a better performance when compared to the LS approach, without DFT estimation. To enhance the performance, DFT based CE is proposed in this article and from the figure we can infer that there is a reduction in MSE significantly. The results suggest the benefit of using DFT based CE methods but this comes at a cost of additional computational complexity.

The received signal constellation of a GFDM system with 16-QAM before and after channel compensation is shown in Figure 8. We can infer from the figure that the data 

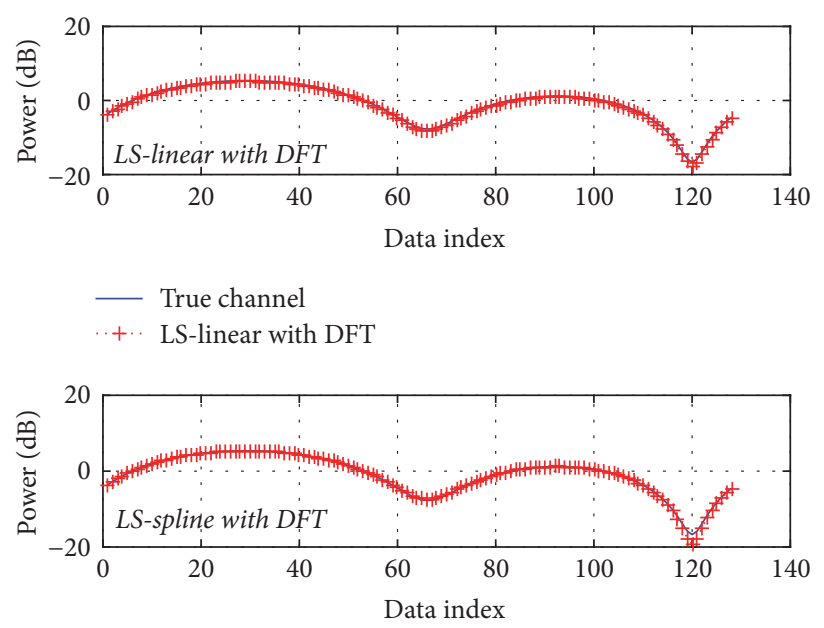

- True channel

-.+. LS-spline with DFT

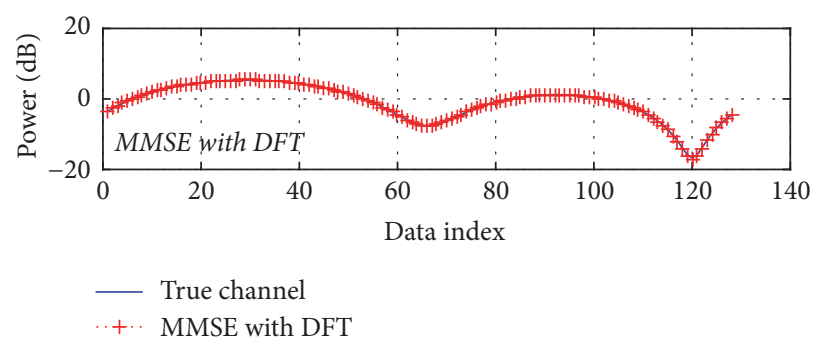

FIGURE 6: DFT based channel estimation.

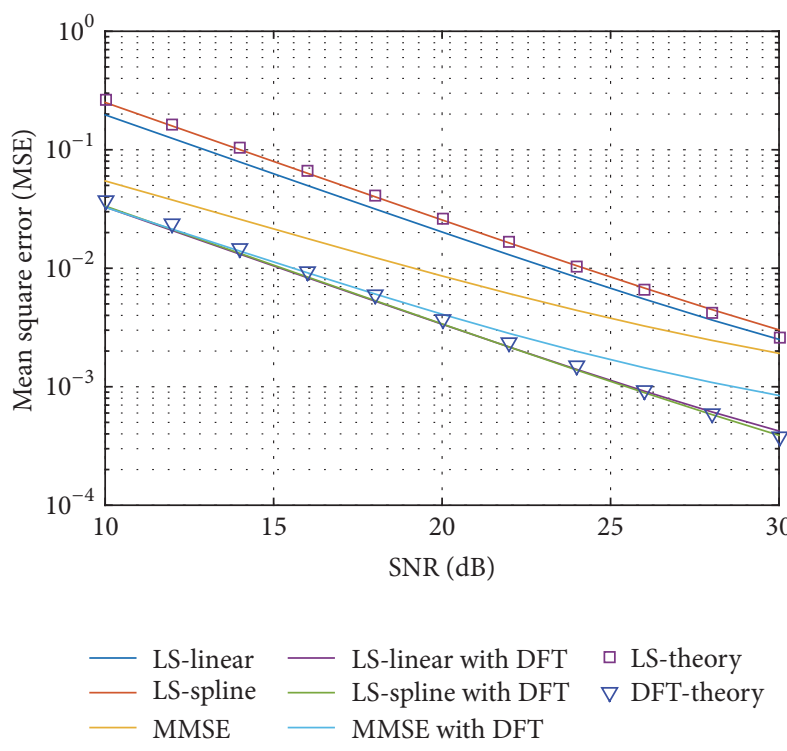

FIGURE 7: Performance evaluation of MGFDM with different channel estimation techniques.

symbols are properly mapped after channel compensation using MMSE channel estimation with DFT technique. From these results it is obvious that DFT based channel estimation methods reduce the MSE at a faster rate.
Lastly, the power spectral densities (PSD) for the various multicarrier techniques are displayed in Figure 9. The simulations are carried out in Matlab and the parameters that are used are given in Table 1 with a sampling frequency of $20 \mathrm{MHz}$ using the pwelch spectral estimation method. From the figure we can observe that the PSD of GFDM has a better spectral efficiency property compared to an OFDM system. Interestingly, the spectra of GFDM using multitapers exhibit the same spectral properties as that of a conventional GFDM system. Thus with the same spectral properties, deeper sidelobes of the pulse shaping filter can be achieved, as discussed earlier.

\section{Conclusion}

In this paper, we have presented channel estimation techniques, namely, LS (with linear and spline interpolation) and MMSE with and without the DFT method.

The channel estimation at the pilot tones and the interpolation of the channel at remaining points are presented. From the simulation results, among the investigated methods, when compared to the LS estimator, the performance of the MMSE estimator is observed to provide a better estimate of the channel response in both cases with and without DFT.

There is an additional computational cost that has to be paid in using DFT based channel estimation. Moreover as we increase the number of data sample points, the complexity of MMSE increases exponentially. Also, the overall efficiency 

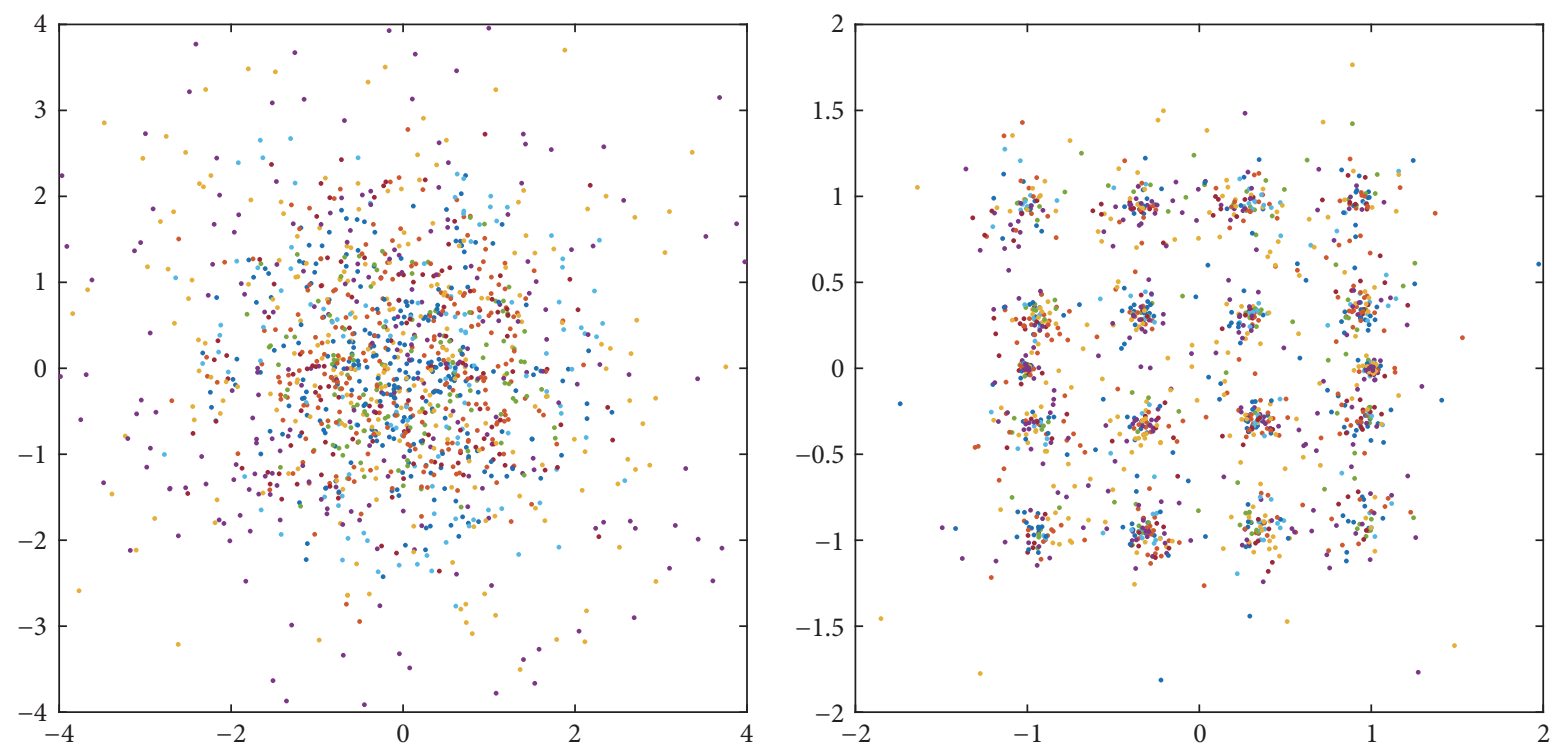

FIGURE 8: Received signal constellation before and after channel compensation.

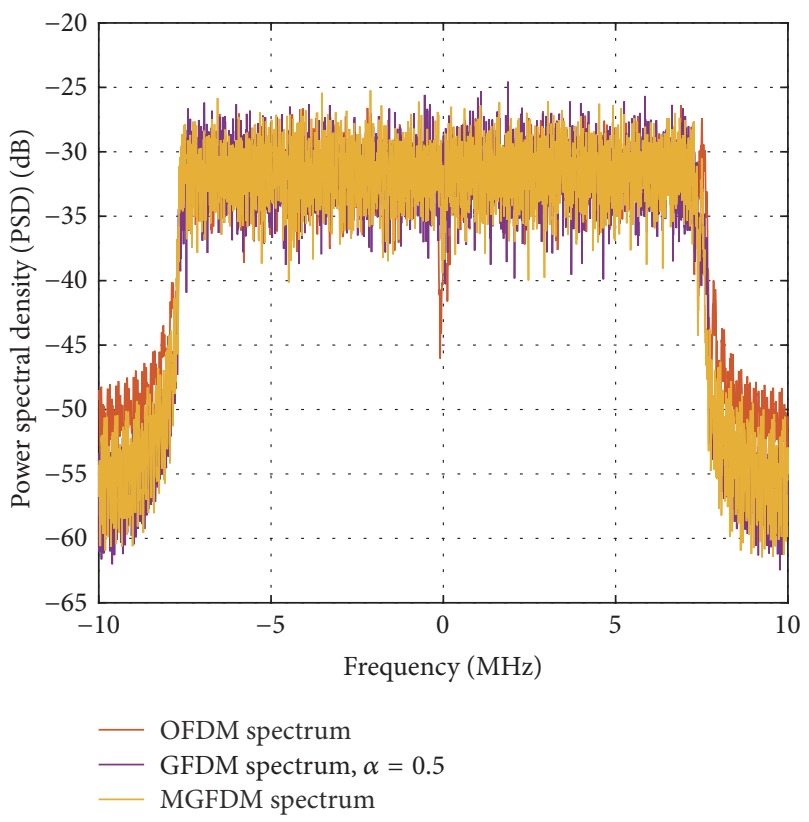

FIGURE 9: Spectrum comparison of OFDM, conventional GFDM with root raised cosine filter of roll-off factor 0.5, and MGFDM systems.

of the system may be reduced due to the addition of the overhead pilot symbols to that of data symbols. Therefore a trade-off exists between better channel estimation system performance and additional complexity of the overall system. This paper addresses the preliminary investigation of channel estimation methods on the novel multicarrier MGFDM system technique. It will be of great interest to implement some of the advanced channel estimation techniques in usage today and propose new such techniques for MGFDM, which is where our future work focuses.

\section{Conflicts of Interest}

The authors declare that they have no conflicts of interest.

\section{References}

[1] A. R. Bahai, B. R. Saltzberg, and M. Ergen, Multi-Carrier Digital Communications: Theory and Applications of OFDM, Springer Science \& Business Media, Berlin, Germany, 2004.

[2] B. Farhang-Boroujeny, "OFDM versus filter bank multicarrier," IEEE Signal Processing Magazine, vol. 28, no. 3, pp. 92-112, 2011.

[3] V. Vakilian, T. Wild, F. Schaich, S. Ten Brink, and J.-F. Frigon, "Universal-filtered multi-carrier technique for wireless systems beyond LTE," in Proceedings of the 2013 IEEE Globecom Workshops, GC Wkshps 2013, pp. 223-228, Atlanta, Ga, USA, December 2013.

[4] N. Michailow, M. Matthe, I. S. Gaspar et al., "Generalized frequency division multiplexing for 5 th generation cellular networks," IEEE Transactions on Communications, vol. 62, no. 9, pp. 3045-3061, 2014.

[5] U. Vilaipornsawai and M. Jia, "Scattered-pilot channel estimation for GFDM," in Proceedings of the 2014 IEEE Wireless Communications and Networking Conference, WCNC 2014, pp. 1053-1058, Istanbul, Turkey, April 2014.

[6] L. Chang, G. Y. Li, J. Li, and R. Li, "Blind parameter estimation of GFDM signals over frequency-selective fading channels," IEEE Transactions on Communications, vol. 64, no. 3, pp. 1120-1131, 2016.

[7] S. K. Bandari, V. V. Mani, and A. Drosopoulos, "Multi-Taper implementation of GFDM," in Proceedings of the 2016 IEEE Wireless Communications and Networking Conference, WCNC 2016, qat, April 2016.

[8] M. Danneberg, N. Michailow, I. Gaspar et al., "Implementation of a 2 by 2 MIMO-GFDM transceiver for robust 5G networks," in Proceedings of the 2015 International Symposium on Wireless Communication Systems (ISWCS), pp. 236-240, Brussels, Belgium, August 2015. 
[9] J.-J. van de Beek, O. Edfors, M. Sandell, S. K. Wilson, and P. O. Borjesson, "On channel estimation in OFDM systems," in Proceedings of the 1995 IEEE 45th Vehicular Technology Conference. Part 2 (of 2), pp. 815-819, July 1995.

[10] F. Tufvesson and T. Maseng, "Pilot assisted channel estimation for OFDM in mobile cellular systems," in Proceedings of the 1997 47th IEEE Vehicular Technology Conference. Part 1 (of 3), pp. 1639-1643, May 1997.

[11] D. Slepian, "Prolate spheroidal wave functions, fourier analysis, and uncertainty-V: the discrete case," Bell System Technical Journal, vol. 57, no. 5, pp. 1371-1430, 1978.

[12] A. Farhang, N. Marchetti, and L. E. Doyle, "Low-complexity modem design for GFDM," IEEE Transactions on Signal Processing, vol. 64, no. 6, pp. 1507-1518, 2016.

[13] M.-H. Hsieh and C.-H. Wei, "Channel estimation for OFDM systems based on comb-type pilot arrangement in frequency selective fading channels," IEEE Transactions on Consumer Electronics, vol. 44, no. 1, pp. 217-225, 1998.

[14] O. Edfors, M. Sandell, J.-J. Van De Beek, S. K. Wilson, and P. O. Börjesson, "Analysis of DFT-based channel estimators for OFDM," Wireless Personal Communications, vol. 12, no. 1, pp. 55-70, 2000.

[15] M. J. F.-G. García, J. M. Páez-Borrallo, and S. Zazo, "DFT-based channel estimation in 2D-pilot-symbol-aided OFDM wireless systems," IEEE Vehicular Technology Conference, vol. 2, no. 53, pp. 810-814, 2001.

[16] Y. Shen and E. Martinez, "Channel estimation in OFDM systems," 2006. 

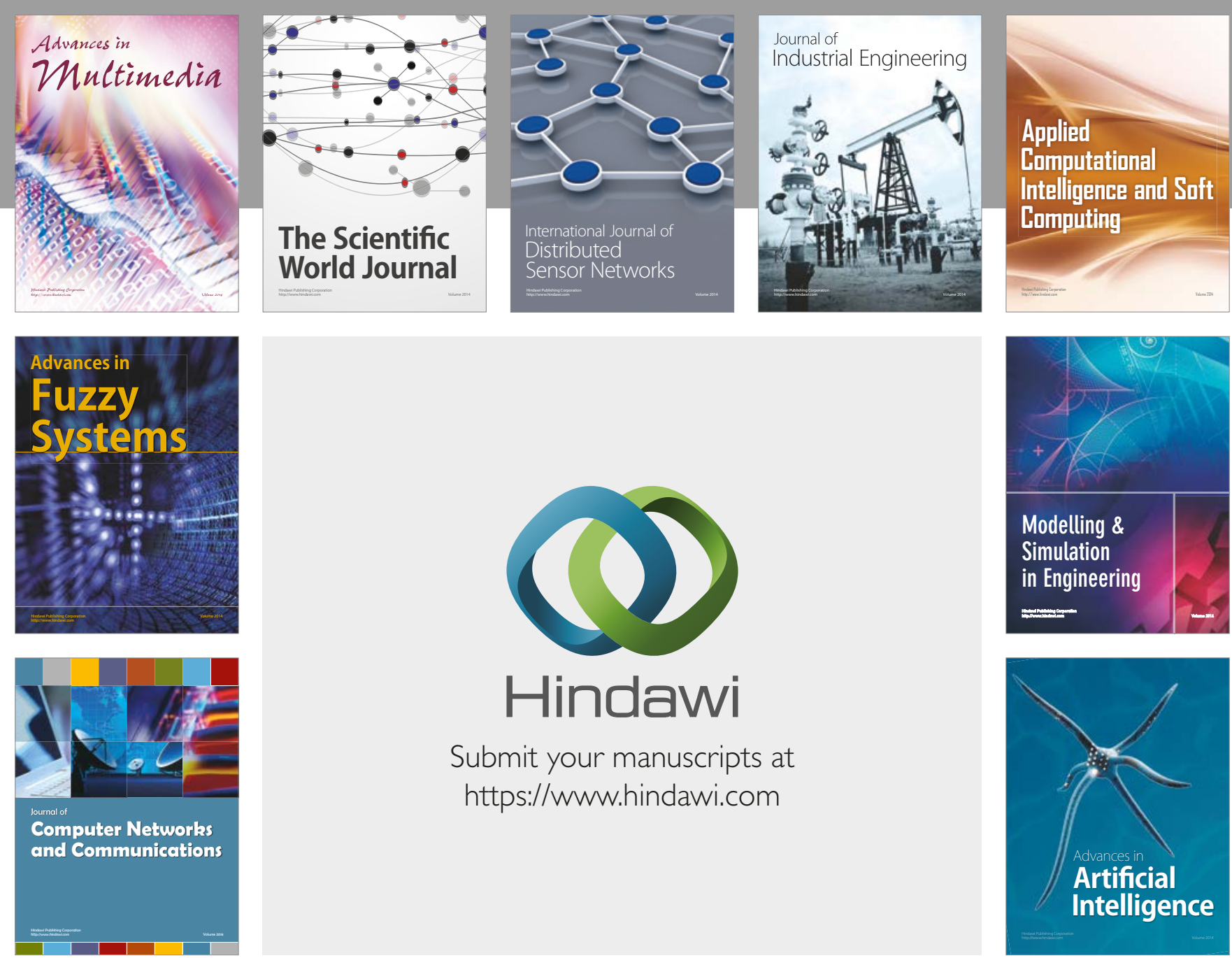

\section{Hindawi}

Submit your manuscripts at

https://www.hindawi.com
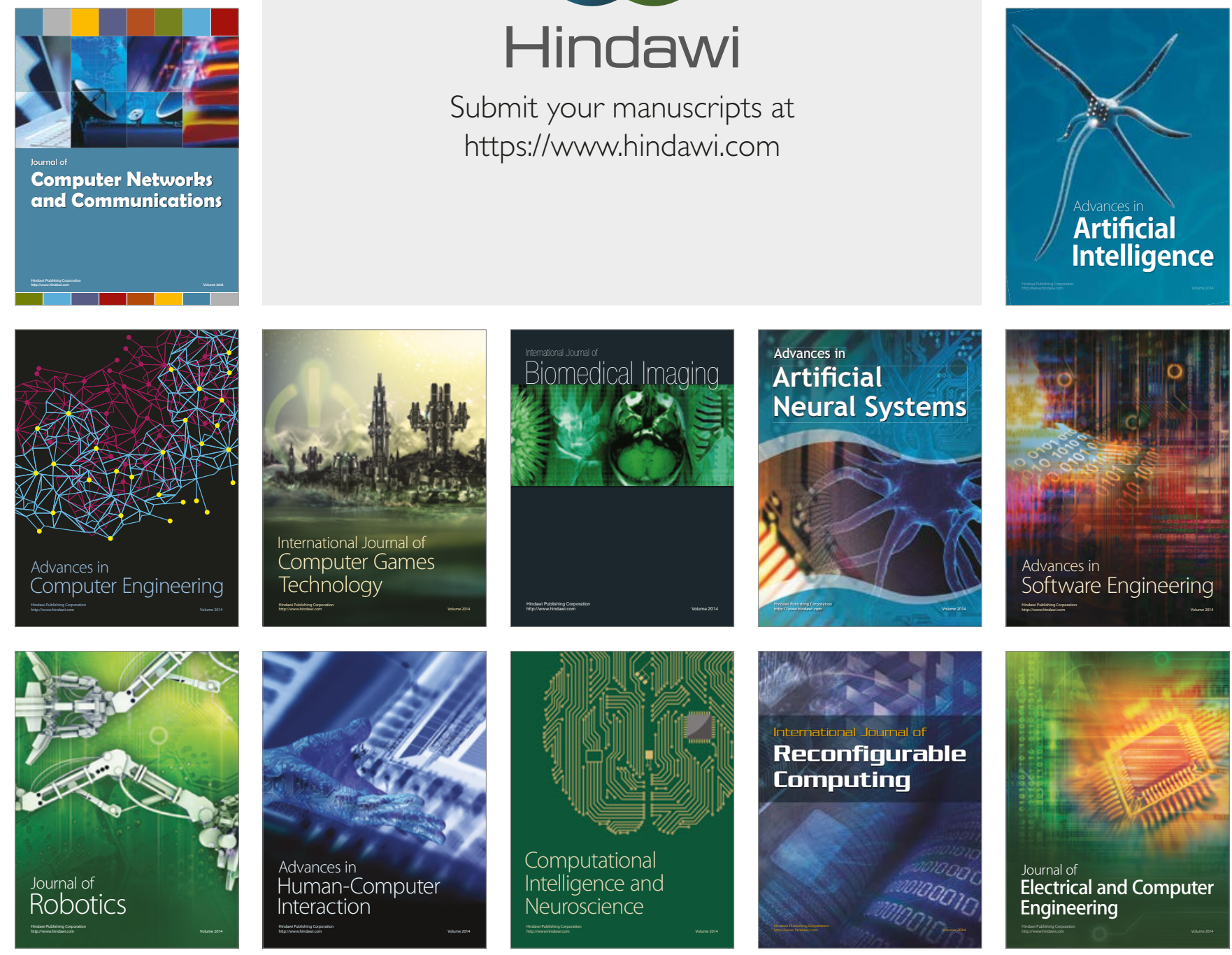